\title{
The Comparison of Candida spp. Colonization on Psoriasis Vulgaris Patient and Control
}

\author{
Amanda Gracia Manuputty ${ }^{1}$, Dwi Murtiastutik ${ }^{1}$, Sawitri ${ }^{1}$, Muhammad Yulianto \\ Listiawan $^{1}$, Rahmadewi ${ }^{1}$, Afif Nurul Hidayati', Pepy Dwi Endraswari ${ }^{2}$, Evy Ervianti ${ }^{1}$ \\ ${ }^{I}$ Department of Dermatology and Venereology, Faculty of Medicine, Universitas Airlangga, Dr. \\ Soetomo General Academic Teaching Hospital, Surabaya, Indonesia \\ ${ }^{2}$ Departemen Clinical Microbiology, Faculty of Medicine, Universitas Airlangga, Dr. Soetomo General \\ Academic Teaching Hospital, Surabaya, Indonesia
}

\begin{abstract}
Background: Psoriasis is an inflammatory, chronic, and recurring skin disease that affects patient's quality of life. Environment, drugs, and infections in the form of antigen and toxin such as Candida spp. are assumed responsible for triggering and exacerbating psoriasis. Purpose: To compare the prevalence of skin colonization of Candida spp. on psoriasis vulgaris patient and control. Methods: This study was observational analytic research involving 33 psoriasis vulgaris patients and 33 healthy individuals. They were matched up based on sex, age, and lesion location. Swab of skin lesions on the Koebner area of both groups was cultured on CHROMagar media. Candida spp. was later identified and the colonization was calculated. Result: Candida spp. was found on $51.5 \%$ of lesions in psoriasis vulgaris patients and $21.2 \%$ in the control group with a significant difference of $\mathrm{p}<0.05$. The average colony number of Candida spp. in psoriasis vulgaris group was lower than the control group with no significant difference of $\mathrm{p}>0.05$. The predominant Candida spp. in the psoriasis vulgaris and control groups was Candida krusei, which were $35.5 \%$ and $85.6 \%$, respectively. No significant difference between sex, age, period of complaints, age onset, degree of severity (Psoriasis Area and Severity Index), and therapy history, with positive culture of Candida spp. Conclusion: There was no difference in the number of Candida spp. colonization in the psoriasis vulgaris group and the control group. However, the culture positivity of Candida spp. in the psoriasis vulgaris patients was higher than in the control group.
\end{abstract}

Keywords: Candida spp., CHROMagar, Psoriasis vulgaris.

Correspondence: Evy Ervianti, Department of Dermatology and Venereology, Faculty of Medicine, Universitas Airlangga/Dr. Soetomo General Academic Teaching Hospital, Jl. Mayjen Prof. Dr. Moestopo No. 6-8 Surabaya 60131, Indonesia. Telephone: +6281-3326815133, e-mail: evy_if@yahoo.co.id.

\section{BACKGROUND}

Psoriasis is an inflammatory, chronic, recurrent skin disease characterized by red plaques, unsharply marginated, and covered with thick squama. It is caused by uncontrolled proliferation and differentiation of keratinocytes in the epidermis. ${ }^{1,2}$ This disease is associated with inflammatory dysregulation of the immune system (immunological processes mediated), genetic, and environmental. Its prevalence ranges from $2 \%-4 \%$ worldwide. Psoriasis vulgaris or plaque type psoriasis is the classic and the most common form, accounting for $85-90 \%$ of all psoriasis cases. ${ }^{2,3}$ Several factors including environment, infections (bacterial, fungal, and viral), individual stress, and drug exposure can provoke and exacerbate psoriasis. ${ }^{4,5}$ Psoriasis, which often recurs and persists in a chronic state, has a negative effect on the patient's quality of life in terms of self confidence, psychological burden (depression), and financial burden. ${ }^{6}$
Over the past few years, studies have suggested that microbial dysbiosis of the skin and mucosa can increase immune response in susceptible hosts, inducing a persistent inflammatory process associated with autoimmune disorders. Some bacteria (Staphylococcus pyogenes, Staphylococcus aureus), fungi (Malassezia and Candida), and viruses (Papillomavirus, Retrovirus) act as superantigens that activate non specific $\mathrm{T}$ cells thus initiating, exacerbating, and maintaining a chronic inflammatory process in skin diseases thus disrupting the patient's quality of life. ${ }^{4}$ In the amino acid sequence, Candida albicans (C. albicans) have an Int1 protein cell wall that shows a part that can directly bound to the class II major histocompatibility complex (MHC) so that it has a superantigen like effect. This superantigen like effect will induce non specific $\mathrm{T}$ lymphocytes without going through the classical antigen process and release excessive proinflammatory cytokines, causing cell damage. $^{7}$ 
Previous studies showed that Candida colonization was found more on the oral mucosa, skin, and gastrointestinal tract of psoriasis patients than healthy people. ${ }^{8}$ In 2014 , Sarvtin et al. reported that the number of colonization of C. albicans was $15 \%$ in psoriasis vulgaris patients and 4 in healthy persons (p $<0.05){ }^{9}$ Also, in 2015, Pezeshkpoor et al. in 2015 reported higher $C$. albicans colonies in oral of psoriasis patients $(46 \%)$ than in healthy persons $(18 \%)$ and on the skin $(2 \%)$ than healthy persons $(0 \%)$ with a significant difference $(\mathrm{p}<0.05) .{ }^{10}$ Underlying mechanisms of the association between Candida spp. colonization and the severity of psoriasis are not fully understood. However, the number of Candida tends to progress along with changes in a compromised immune system. Therefore, in conditions of chronic inflammatory skin disorders such as atopic dermatitis and psoriasis, the prevalence of Candida spp. colonization would increase. Moreover, the pharmacological intervention for psoriasis patients is in the form of corticosteroid and immunosuppressive agents such as methotrexate, inhibitor tumor necrosis factor-alfa (TNF- $\alpha$ ), and interleukin-17 (IL-17) could affect the response of the antifungal immune. ${ }^{1,11}$ As a result, the risk of Candida spp. superficial infection will also increase in those suffering psoriasis. ${ }^{12}$ This was based on previous research, comparing the colonization of Candida spp. between psoriasis vulgaris patients and control. Such condition may act as a triggering factor and exacerbation of psoriasis.

\section{METHODS}

This was observational analytic research aimed to determine the differences between Candida spp. colonization on psoriasis vulgaris patients and healthy persons. We used a pair of samples, involving 35 psoriasis vulgaris patients and 35 healthy individuals. This research took place at the Dermatology and Venereology Outpatient Clinic, Dr. Soetomo General Academic Teaching Hospital Surabaya from JanuaryMarch 2020. Psoriasis vulgaris patients, aged 18-65 years, diagnosed through clinical and histopathological examinations and signed informed consent. The control group consisted of healthy individuals in which age and sex matched with psoriasis vulgaris patients. The rejection criteria were patients taking oral antifungals in the last 1 month or topical antifungals in the last 2 weeks, pregnant, and diagnosed with diabetes mellitus, malignancy, or Human Immunodeficiency Virus (HIV)/ acquired immune deficiency syndrome (AIDS). We used consecutive sampling, which means selecting subjects per the acceptance criteria.
Demographic data and medical conditions of the sample included age, sex, length of the complaint, age of onset, severity, and medication history. Severity of the disease is measured based on the Psoriasis Area and Severity Index (PASI) score. The data collected from anamnesis, physical examination, skin specimens of psoriatic lesion from psoriasis patient and healthy skin from a control subject, specimens were growth the culture medium, and Candida colonies were counted. The culture medium used was CHROMagar Candida. Skin specimens were collected using a skin swab of 3 $\mathrm{x} 3 \mathrm{~cm}^{2}$ in the Koebner area with a sterile cotton swab soaked in $1 \mathrm{ml}$ of $0.9 \%$ normal saline solution, both in psoriasis vulgaris patients and healthy individuals. Swab results were put into the culture medium and allowed Candida to grow for $36-48$ hours at $37^{\circ} \mathrm{C}$. The number of colonies was counted and the Candida spp. identification through color changes produced by colony growth. The Candida culture examination was carried out at the Microbiology Laboratory of Dr. Soetomo General Academic Teaching Hospital Surabaya. The collected data were analyzed using the Statistical Package for Social Science (SPSS) software release version 17. Comparative tests were carried out using the chi square test and the t test, while the Shapiro Wilk test was used for the normality test. Ethical clearance was obtained from the Ethics Committee of Dr. Soetomo General Academic Teaching Hospital Surabaya before this research was conducted, Number 1652/KEPK/XI/2019.

\section{RESULT}

Thirty-three patients were diagnosed with psoriasis vulgaris clinically and histopathologically. Each group consisted of 20 males $(60.6 \%)$ and 13 females $(39.4 \%)$ with a ratio of $1.5: 1$. There were 11 individuals $(33.3 \%)$ in the $45-55$ years age group and 4 individuals $(12.1 \%)$ in the $18-25$ years age group (p $=1.000)$. The mean age of each group was $43.33+$ 11.73 years. The statistical test results showed a homogeneous distribution in sex and age between the psoriasis vulgaris group and the control group (Table $1)$.

We found more Candida spp. positivity in females $(53.8 \%)$ than males $(50.0 \%)$. The statistical test results showed a difference between Candida spp. culture between both sexes ( $\mathrm{p}>0.05$ ). We found $100 \%$ Candida spp. positivity in the 18-25 and 55-65 years age group of the psoriasis vulgaris group. However, there was no significant difference in culture positivity based on age in the psoriasis vulgaris group $(\mathrm{p}=0.711)$ (Table 2). 
Table 1. The distribution of sex and age on the psoriasis vulgaris and control groups

\begin{tabular}{|c|c|c|c|c|}
\hline Variables & $\begin{array}{l}\text { Psoriasis vulgaris } \\
\quad(\mathrm{n}=33)(\%)\end{array}$ & $\begin{array}{c}\text { Control } \\
(\mathrm{n}=33)(\%)\end{array}$ & $\begin{array}{c}\text { Quantity } \\
(\mathrm{n}=66)(\%)\end{array}$ & p-value \\
\hline Sex & & & & 1.000 \\
\hline Male & $20(60.6)$ & $20(60.6)$ & $40(60.6)$ & \\
\hline - Female & $13(39.4)$ & $13(39.4)$ & $26(39.4)$ & \\
\hline Age (years) & & & & 0.959 \\
\hline$-\quad 18-25$ & $4(12.1)$ & $4(12.1)$ & $8(12.1)$ & \\
\hline$-\quad 26-35$ & $5(15.2)$ & $5(15.2)$ & $10(15.2)$ & \\
\hline$-\quad 36-45$ & $7(21.2)$ & $7(21.2)$ & $14(21.2)$ & \\
\hline - $46-55$ & $11(33.3)$ & $10(30.3)$ & $21(31.8)$ & \\
\hline$-\quad 56-65$ & $6(18.2)$ & $7(21.2)$ & $13(19.7)$ & \\
\hline
\end{tabular}

Table 2. The distribution of Candida spp. culture positivity proportion on psoriasis vulgaris group based on sex and age

\begin{tabular}{|c|c|c|c|c|c|}
\hline \multirow{2}{*}{\multicolumn{2}{|c|}{ Variables }} & \multicolumn{2}{|c|}{ Psoriasis vulgaris } & \multirow{2}{*}{$\begin{array}{c}\text { Quantity } \\
n=33(\%)\end{array}$} & \multirow[t]{2}{*}{$\mathrm{p}$-value } \\
\hline & & $\begin{array}{l}\text { Candida spp. } \\
\text { Culture positive } \\
(\mathrm{n}=17)(\%)\end{array}$ & $\begin{array}{l}\text { Candida spp. } \\
\text { Culture negative } \\
(\mathrm{n}=16)(\%)\end{array}$ & & \\
\hline \multicolumn{5}{|l|}{ Sex } & \multirow[t]{3}{*}{1.000} \\
\hline- & Male & $10(50.0)$ & $10(50.0)$ & $20(60.6)$ & \\
\hline- & Female & $7(53.8)$ & $6(46.2)$ & $13(39.4)$ & \\
\hline \multicolumn{4}{|c|}{ Age (years) } & & \multirow[t]{6}{*}{0.711} \\
\hline- & 18-25 (Late adolescent) & $4(100)$ & 0 & $4(12.1)$ & \\
\hline - & 26-35 (Early adult) & $2(40.0)$ & $3(60.0)$ & $5(15.2)$ & \\
\hline- & 36-45 (Late adult) & $2(28.6)$ & $5(71.4)$ & $7(21.2)$ & \\
\hline- & 46-55 (Early elderly) & $3(27.3)$ & $8(72.7)$ & $11(33.3)$ & \\
\hline- & 56-65 (Late elderly) & $6(100)$ & 0 & $6(18.2)$ & \\
\hline
\end{tabular}

The highest prevalence of illness duration in psoriasis vulgaris patients was $>10$ years $(51.5 \%)$ and the mean duration of complaints in psoriasis vulgaris patients was $11.73 \pm 8.73$. The results of Candida spp. positivity based on the illness duration were found for $<5$ years $(65.2 \%), 5-10$ years $(65.2 \%)$, and $>10$ years $(41.2 \%)$. However, statistically, there was no significant difference $(p=0.274)$. In this research, the age of disease onset in the psoriasis vulgaris group was mostly $<30$ years $(54.5 \%)$ and the mean age of onset was $31.15 \pm 10.93$. The results of Candida spp. culture positivity on age of onset were $\geq 30$ years $(53.3 \%)$ and $<30$ years $(50 \%)$ but not statistically significant $(\mathrm{p}=$ 1.000) (Table 3).

We found 20 patients $(60.6 \%)$ get into the most severe category with PASI $>10$ and the average PASI score in psoriasis vulgaris patients was 13.19 \pm 8.71 . Ten psoriasis vulgaris patients $(50 \%)$ with severe severity (PASI $>10$ ) had positive culture results for Candida spp., while 10 patients $(50 \%)$ had negative culture results. In moderate (PASI 5-10) and mild $(\mathrm{PASI}<5)$ severity, 5 patients $(83.3 \%)$ and 2 patients (28.6\%) had positive cultures for Candida spp., respectively. However, there was no significant difference in the degrees of severity $(\mathrm{p}=0.789)$ (Table 3 ). Positive culture result means positive for Candida spp. based on the treatment history, all of the methotrexate, topical corticosteroids, and narrowbandultraviolet B (NB-UVB) phototherapy did not have a significant statistical difference $(p>0.05)$ (Table 4).

Of the 33 psoriasis vulgaris patients, positive cultures of Candida spp. were found in $51.5 \%$ of psoriasis vulgaris patients and only $21.2 \%$ positive cultures were found in the control group with statistically significant differences $(\mathrm{p}=0.021)$ (Table 5). The Odds Ratio of Candida spp. colonization on the skin of psoriasis vulgaris patients and the control group was 3.94. The mean colony count of Candida spp. in the psoriasis vulgaris group was smaller than the control group, which was $6.71 \pm 18.850$ and $10.29 \pm$ 9.759, respectively. The Mann-Whitney test result showed that the mean value of Candida spp. of the two groups did not differ significantly $(\mathrm{p}=0.226)$ (Table $5)$.

Of the 25 study subjects with Candida colony growth, the identification of $C$. albicans was found in 5 psoriasis vulgaris patients $29.4 \%, C$. krusei $35.3 \%, C$. glabrata $5.8 \%$, the remaining was a mixture of two 
types of Candida, namely C. albicans and C. krusei $23.5 \%, C$. krusei and C. tropicalis 5.8\%. In the control group there were no $C$. albicans, but only $85.7 \% C$. krusei, 14.3\% C. albicans, and 14.3\% C. krusei (Table 6).

Table 3. The distribution of Candida spp. culture positivity proportion on psoriasis vulgaris group based on the duration of psoriasis, onset of psoriasis, and PASI score

\begin{tabular}{|c|c|c|c|c|}
\hline \multirow[t]{2}{*}{ Variables } & \multicolumn{2}{|c|}{ Psoriasis vulgaris } & \multirow{2}{*}{$\begin{array}{c}\text { Quantity } \\
n=33(\%)\end{array}$} & \multirow[t]{2}{*}{$\mathrm{p}$-value } \\
\hline & $\begin{array}{l}\text { Candida spp. } \\
\text { Culture positive } \\
(\mathrm{n}=17)(\%)\end{array}$ & $\begin{array}{l}\text { Candida spp. } \\
\text { Culture negative } \\
(\mathrm{n}=16)(\%)\end{array}$ & & \\
\hline Psoriasis duration & & & & 0.253 \\
\hline$-\quad<5$ years & $5(65.2)$ & $3(37.5)$ & $8(24.2)$ & \\
\hline - $\quad 5-10$ years & $5(65.2)$ & $3(37.5)$ & $8(24.2)$ & \\
\hline$-\quad>10$ years & $7(41.2)$ & $10(58.8)$ & $17(51.5)$ & \\
\hline Onset of psoriasis & & & & 1.000 \\
\hline$-\quad<30$ years & $9(50.0)$ & $9(50.0)$ & $18(54.5)$ & \\
\hline$-\quad \geq 30$ years & $8(53.3)$ & $7(46.7)$ & $15(45.5)$ & \\
\hline Psoriasis severity (PASI Score) & & & & 0.789 \\
\hline - $\quad$ Mild (PASI < 5) & $2(28.6)$ & $5(71.4)$ & $7(21.2)$ & \\
\hline - $\quad$ Moderate (PASI 5-10) & $5(83.3)$ & $1(16.7)$ & $6(18.2)$ & \\
\hline - $\quad$ High $($ PASI $>10)$ & $10(50.0)$ & $10(50.0)$ & $20(60.6)$ & \\
\hline
\end{tabular}

PASI=Psoriasis Area and Severity Index

Table 4. The distribution of Candida spp. culture positivity proportion on psoriasis vulgaris group based on treatment modality

\begin{tabular}{|c|c|c|c|c|}
\hline \multirow[t]{2}{*}{ Variables } & \multicolumn{2}{|c|}{ Psoriasis vulgaris } & \multirow{2}{*}{$\begin{array}{c}\text { Quantity } \\
\mathrm{n}=33(\%)\end{array}$} & \multirow[t]{2}{*}{ p-value } \\
\hline & $\begin{array}{l}\text { Candida spp. } \\
\text { Culture positive } \\
(\mathrm{n}=17)(\%)\end{array}$ & $\begin{array}{l}\text { Candida spp. Culture negative } \\
\qquad(\mathrm{n}=16)(\%)\end{array}$ & & \\
\hline \multicolumn{5}{|l|}{ Treatment modality } \\
\hline - Systemic Methotrexate & & & & 0.708 \\
\hline - Yes & $11(47.8)$ & $12(52.2)$ & $23(69.7)$ & \\
\hline$-\quad$ No & $6(60.0)$ & $4(40.0)$ & $10(30.3)$ & \\
\hline - Topical Corticosteroid & & & & 1.000 \\
\hline - Yes & $16(51.6)$ & $15(48.4)$ & $31(93.9)$ & \\
\hline - $\quad$ No & $1(50.0)$ & $1(50.0)$ & $2(6.1)$ & \\
\hline - NB-UVB Phototherapy & & & & 0.199 \\
\hline$-\quad$ Yes & $8(40.0)$ & $12(60.0)$ & $20(60.6)$ & \\
\hline - No & $9(69.2)$ & $4(30.8)$ & $13(39.4)$ & \\
\hline
\end{tabular}

NB-UVB=Narrowband-ultraviolet B

Table 5. Distribution of culture proportion and Candida spp. on psoriasis vulgaris and control groups

\begin{tabular}{lcccc}
\multicolumn{1}{c}{$\begin{array}{c}\text { Candida } \text { spp. } \\
\text { culture result }\end{array}$} & $\begin{array}{c}\text { Psoriasis vulgaris } \\
(\mathrm{n}=33)\end{array}$ & $\begin{array}{c}\text { Control } \\
(\mathrm{n}=33)\end{array}$ & p-value & OR \\
\hline Positive & $17(51.5)$ & $7(21.2)$ & 0.021 & 3.94
\end{tabular}

$\mathrm{OR}=$ Odd ratio 
Table 6. Distribution of Candida spp. on psoriasis vulgaris and control groups with positive culture

\begin{tabular}{lcc}
\hline \multicolumn{1}{c}{ Candida spp. } & $\begin{array}{c}\text { Psoriasis vulgaris } \\
(\mathrm{n}=17)(\%)\end{array}$ & $\begin{array}{c}\text { Control } \\
(\mathrm{n}=7)(\%)\end{array}$ \\
\hline C. albicans & $5(29.4)$ & 0 \\
\hline C. krusei & $6(35.3)$ & $6(85.7)$ \\
\hline C. glabrata & $1(5.8)$ & 0 \\
\hline C. albicans + C. krusei & $4(23.5)$ & $1(14.3)$ \\
\hline C. krusei + C. tropicalis & $1(5.8)$ & 0 \\
\hline
\end{tabular}

\section{DISCUSSION}

This research found that there were more male patients $(60.6 \%)$ than female patients $(39.4 \%)$ with a ratio of $1.5: 1$. This is in accordance with the study of Ogawa et al. in 2017 in Japan, reporting that $71.5 \%$ of the participants were males and $28.5 \%$ were females. ${ }^{13}$ In 2018, Chiu et al. reported the 1: 1.4 sex ratio of males and females. ${ }^{14}$ Most of the patients were under the 46-55 years age group, which was 11 people $(33.3 \%)$. The range of age in the psoriasis vulgaris group was $43.33 \pm 11.96$ years and $43.48 \pm 11.80$ years in the control group. In 2016, Chularojanamontri et al. reported that the mean age of the psoriasis vulgaris group was $46.0 \pm 14.6$ years and $45.5 \pm 14.6$ years in the control group. ${ }^{15}$ Ogawa et al. reported an increase in the distribution of psoriasis incidence persons aged $40-49$ years. $^{13}$

In this research, we found higher Candida spp. positivite culture result in female psoriasis vulgaris patients than in the male with insignificant difference $(\mathrm{p}=1.000)$. In 2017, Rafat et al. reported that 51.2\% Candida spp. colonization in male participants and 48.7\% female participants, with statistically insignificant difference based on sex. ${ }^{16}$ Furthermore, Somerville stated that Candida colonization is more common in females than males. ${ }^{17}$ Multiple factors, including sociodemographic patterns, economic levels, and lifestyles might affect the research results.

Based on age, the Candida spp. positive culture results were mostly found in the 18-25 years age group $100 \%$ and 56-65 years age group $100 \%$, with no significant difference statistically $(\mathrm{p}=0.711)$. Lee et al. reported that trans epidermal water loss (TEWL) increased in psoriasis vulgaris patients and psoriasis vulgaris patients had drier skin compared to the control. ${ }^{18}$ This indicates that the skin condition of the psoriasis vulgaris group was drier than healthy individuals. In 2017, Rafat et al. reported that Candida colonization found the most in the $>60$ years age group $(51.2 \%){ }^{16}$ The higher prevalence of Candida isolation in elderly might due to a drier skin that corresponds to $\mathrm{pH}$ elevation, resulting in a more alkaline skin. This decreases the ability of an impaired immune system in this age group. The low $\mathrm{pH}$ condition of the skin helps to stabilize the skin's normal flora and adhere to the skin, while an alkaline environment promotes the spread and growth of pathogenic bacteria from the skin.

Psoriasis vulgaris is a chronic and residual inflammatory skin disease. The mean duration of complaints experienced by the psoriasis vulgaris group was $11.73 \pm 8.73$, with the highest prevalence of complaint length was $>10$ years as much as $51.5 \%$. Positivity results of Candida spp. culture in psoriasis vulgaris patients were found mostly in patients with a duration of complaint $<5$ years and $5-10$ years $(65.2 \%)$ compared to $>10$ years $(41.2 \%$, ) but the difference between the duration of complaint in three groups was not significant $(\mathrm{p}=0.253)$. Two studies found that oral Candida colonization was found more common in patients with a complaint duration of $\geq 10$ years $32 \%$ and $69.4 \%$ compared to $<10$ years, which were $26 \%$ and $68.6 \%$, respectively. Also, no significant difference between the two groups $(\mathrm{p}=0.65$ and $\mathrm{p}=$ 0.93). ${ }^{15,19}$ Positivity culture of Candida spp. based on the age onset, mostly were $\geq 30$ years $(53.3 \%)$ than that of $<30$ years $(\mathrm{p}=1.000)$. Research by Bedair et al. found the medium of Candida in the mouth was more common at late onset $\geq 30$ years compared to early onset $<30$ years with a significant statistical difference $(p=0.002) .{ }^{19}$ Based on the available data, the latter observation could not be explained.

In this research, Candida spp. was mostly found on psoriasis vulgaris patients with moderate severity (PASI 5-10) (83.3\%), compared to mild and high degrees of severity with no statistically significant difference $(p=0.789)$. Some studies have not found stronger evidence of Candida colonization in the oral cavity, intestine, and skin, with psoriasis $(p>0.05) .{ }^{9,20}$ Study by Sarvtin et al. on anti Candida antibodies detection reported no significant relationship between PASI score, carriage of Candida spp., and antibody levels in patients with psoriasis. ${ }^{9}$ Psoriasis Area and Severity Index was not an adequate parameter to describe the characteristic of psoriasis vulgaris patients. This led to a suggestion that the severity of psoriasis vulgaris, as measured by the PASI score, only reflects the current status of the disease. Psoriasis vulgaris is a chronic inflammatory process with acute 
attacks. Therefore, the degree of severity as measured by the PASI score may not be related to the positivity of the Candida culture, as suspected, due to one of the causes and triggers of exacerbation of psoriasis vulgaris.

Eleven patients $(47.8 \%)$ who received systemic methotrexate monotherapy had positive culture results for Candida spp. $(\mathrm{p}=0.708)$, while 16 patients $(51.6 \%)$ who received topical corticosteroid therapy had positive culture results for Candida spp. $(\mathrm{p}=1.000)$. Eight patients $(40.0 \%)$ who received NB-UVB phototherapy had positive culture results $(p=0.199)$. There was no statistically significant difference in culture positivity of Candida spp. based on the three types of therapy received by the patients. Several studies suggest an increase in Candida colonization occurs in patients with psoriasis vulgaris and atopic dermatitis. In addition to increased colonization, the risk of superficial Candida infections increases for psoriasis vulgaris patients. This relates to the treatment received by the patient, namely corticosteroids or new immunosuppressive agents such as tumor necrosis factor-alpha (TNF- $\alpha)$ or interleukin-17 (IL-17) inhibitors, because these pharmacological interventions specifically affect the antifungal immune response. ${ }^{11}$ Methotrexate and topical corticosteroid therapy suppress IL-22 and IL-17, respectively, so as not to induce $\beta$-defensin production by epithelial cells. Beta-defensin is a cationic, amphilic antimicrobial peptide of 12-50 amino acids that acts as an antifungal that restricts the overgrowth of Candida colonization. ${ }^{12}$

Positive culture results for Candida spp. were found in $51.5 \%$ of the psoriasis vulgaris patients, whereas only $29.2 \%$ of the control group had positive culture results for Candida spp. with a significant difference of $(\mathrm{p}=0.021)$. Sarvatin et al. found a positive medium of Candida spp. which was $15 \%$ on the skin in the psoriasis vulgaris group and $4 \%$ in the control group. ${ }^{9}$ In 2015, Pezeshkpoor et al. reported that Candida spp. culture positivity was low in skin swab, which was only $2 \%$, compared to the culture positivity of saliva swab, which had high positive culture results in the psoriasis vulgaris group (46\%) and $18 \%$ positive culture results in the control group. ${ }^{21}$

This can be caused by differences in sampling criteria. In our research, we involved patients who received either methotrexate or topical steroid therapy. Psoriasis occurs with increased activation and increased maturation of dendritic cells and keratinocytes induced by increased interferon (IFN)- $\gamma$ and TNF- $\alpha$ as well as increased hyperproliferation and abnormal differentiation of keratinocytes induced by increased IL-23, IL-17, and IL-22 immune responses. The systemic immunosuppressive agent used to treat psoriasis has the key immunological inhibition in lowering IL-22 levels and corticosteroids suppress IL17 production. The suppression of IL-17 and IL-22 has an important role in the phagocytosis mechanism of Candida spp., especially in C. albicans. Besides, a highly humid environment and a higher temperature can increase Candida proliferation, thereby changing its nature to become a pathogen. ${ }^{12}$

This research also showed the odds ratio result of 3.94, where the risk of Candida cultures positivity on the skin of psoriasis vulgaris patients was 3.94 times greater than that of the control group. The metaanalysis study was based on several studies found that colonization of Candida spp. was not significant between psoriasis and control groups with odds ratio of $1.14,(\mathrm{p}=0.001) .{ }^{21}$ Pezeshkpoor et al. reported that the incidence of Candida in the saliva of psoriasis vulgaris patients was 2.5 times higher than that of the healthy group. ${ }^{10}$ A study by Henseler on the medical records of 44,695 dermatology patients concluded that psoriasis patients had a 1.3-1.6 higher relative risk of developing mucocutaneous candidiasis than the healthy group. ${ }^{22} \mathrm{~A}$ study on the medical records of 3,006 psoriasis patients and 1,808 atopic dermatitis patients in Kiel, Germany found that Candida skin infections were found in psoriasis patients more frequently than in controls. However, there was no correlation between Candida skin infections and detected psoriasis activity. ${ }^{23}$

Although only a few previous studies have presented data on the colonization of Candida spp. in psoriasis vulgaris patients in comparison to the control group, they still show that the prevalence of colonization of Candida spp. in psoriasis vulgaris patients was higher than in controls. This may explain that Candida spp. colonization is not the only factor that may trigger and exacerbate psoriasis vulgaris but rather related to the ability of Candida spp. to become superantigen that can directly bound to the class II $\mathrm{MCH}$, induce nonspecific $\mathrm{T}$ lymphocytes (variable $\beta$ on TCR or T Cell Receptors (TCR) which act as antigen receptors) without antigen presenting cell (APC) intermediaries and release excess proinflammatory cytokines, triggering and aggravating the psoriasis vulgaris conditions.

This study found that $C$. non albicans was predominantly found both in psoriasis and control groups. This study also in line with a study by Ovicinakortovic who found that the majority of $C$. non albicans composition of psoriasis vulgaris patient group and psoriasis was $18.3 \% .{ }^{24}$ Within the last two decades, the incidence of non albicans species has been increasing, especially in immunocompromised subjects. ${ }^{25}$ Moreover, other factors such as prematurity, wide spectrum antibiotic consumption, and elderly were 
suspected to be related to an increased incidence in non albicans species. ${ }^{26}$

In conclusion, there was no difference in the number of Candida spp. colonization in the psoriasis vulgaris group and the control group. However, the culture positivity for Candida spp. on the skin of psoriasis vulgaris patients was higher than the control group.

\section{REFERENCES}

1. Rendon A, Schäkel K. Psoriasis pathogenesis and treatment. Int J Mol Sci 2019; 20(1475):1-28.

2. Gudjonsson JE, Elder JT. Psoriasis. In: Kang S, Amagai M, Bruckner A, Enk AH, Margolis DJ, McMichael AJ, et al., editors. Fitzpatrick's Dermatology. New York: McGraw-Hill Education; 2019.p.457-97.

3. Meglio P Di, Villanova F, Nestle FO. Psoriasis. Cold Spring Harb Perspect Med 2014; 4:1-31.

4. Kamiya K, Kishimoto M, Sugai J, Komine M, Ohtsuki M. Risk factors for the development of psoriasis. Int J Mol Sci 2019; 20(18):1-14.

5. Lewis DJ, Chan WH, Hinojosa T, Hsu S, Feldman SR. Mechanisms of microbial pathogenesis and the role of the skin microbiome in psoriasis: A review. Clin Dermatol 2019; 37(2):160-6.

6. Nazik H, Nazik S, Gul FC. Body image, selfesteem, and quality of life in patients with psoriasis. Indian Dermatol Online J 2017; 8(5):343-6.

7. Devore-Carter D, Kar S, Vellucci V, Bhattacherjee V, Domanski P, Hostetter MK. Superantigen-like effects of a Candida albicans polypeptide. J Infect Dis 2008;197(7): 981-9.

8. Sepahi S, Riahi-Zanjani B, Ghorani-Azam A. The role of Candida albicans in the pathogenesis of psoriasis vulgaris: a systematic literature review. Rev Clin Med 2016; 3(3):122-7.

9. Sarvtin MT, Shokohi T, Hajheydari Z, Yazdani J, Hedayati MT. Evaluation of candidal colonization and specific humoral responses against Candida albicans in patients with psoriasis. Int J Dermatol 2014; 53(12):e555-60.

10. Pezeshkpoor F, Yazdanpanah MJ, Family SZ, Sepahi S. Prevalence of Candida in saliva and skin lesions of psoriasis vulgaris patients. J Mycol Res 2015; 2(1):9-14.

11. Kühbacher A, Burger-kentischer A, Rupp S. Interaction of Candida species with the skin. Microorganisms 2017; 5(2):1-12.

12. van der Meer JWM, van de Veerdonk FL, Joosten LAB, Kullberg BJ, Netea MG. Severe Candida spp. infections: New insights into natural immunity. Int J Antimicrob Agents 2010;
36:S58-62.

13. Ogawa E, Okuyama R, Seki T, Kobayashi A, Oiso N, Muto M, et al. Epidemiological survey of patients with psoriasis in Matsumoto city, Nagano Prefecture, Japan. J Dermatol 2018; 45(3):314-7.

14. Chiu HY, Wang TS, Chen PH, Hsu SH, Tsai YC, Tsai TF. Psoriasis in Taiwan: From epidemiology to new treatments. Dermatologica Sin 2018; 36(3):115-23.

15. Chularojanamontri L, Wongpraparut C, Tuchinda P, Winayanuwattikun $\mathrm{W}$, Boonyasiri A, Kulthanan K, et al. Oral Candida colonization in Thai patients with psoriasis. J Med Assoc Thail 2016; 99(1):84-7.

16. Rafat Z, Hashemi SJ, Ahamdikia K, Daie Ghazvini R, Bazvandi F. Study of skin and nail Candida species as a normal flora based on age groups in healthy persons in Tehran-Iran. J Mycol Med 2017; 27(4):501-5.

17. Somerville DA. The normal flora of the skin in different age groups. $\mathrm{Br}$ J Dermatol 1969; 81(4):248-58.

18. Lee Y, Je Y, Lee S, Li ZJ, Choi D, Kwon Y, et al. Changes in transepidermal water loss and skin hydration according to expression of aquaporin-3 in psoriasis. Ann Dermatol 2012; 24(2):168-74.

19. Bedair AA, Darwazeh AMG, Al-Aboosi MM. Oral Candida colonization and candidiasis in patients with psoriasis. Oral Surg Oral Med Oral Pathol Oral Radiol 2012; 114(5):610-5.

20. Waldman A, Gilhar A, Duek L, Berdicevsky I. Incidence of Candida in psoriasis a study on the fungal flora of psoriatic patients. Mycoses 2001; 44:77-81.

21. Pietrzak A, Grywalska E, Socha M, Roliński J, Franciszkiewicz-Pietrzak K, Rudnicka L, et al. Prevalence and possible role of Candida species in patients with psoriasis: A systematic review and meta-analysis. Hindawi Mediat Inflamm 2018; (Article ID 9602362):1-7.

22. Henseler T. Mucocutaneous candidiasis in patients with skin diseases. Mycoses 1995; 38(Suppl 1):7-13.

23. Henseler T, Tausch I. Mycoses in patients with psoriasis or atopic dermatitis. Mycoses 1997; 40(Suppl 1):22-8.

24. Ovčina-Kurtović N, Kasumagić-Halilović E, Helppikangans H, Begić J. Prevalence of Candida species in patients with psoriasis. Acta Dermatovenerologica Croat 2016; 24(3):209-13.

25. Papon N, Courdavault V, Clastre M, Bennett RJ. Emerging and emerged pathogenic Candida species: Beyond the Candida albicans paradigm. 
PLoS Pathog 2013; 9(9):e1003550.

26. Sardi JCO, Scorzoni L, Bernardi T, FuscoAlmeida AM, Mendes Giannini MJS. Candida species: Current epidemiology, pathogenicity, biofilm formation, natural antifungal products and new therapeutic options. J Med Microbiol 2013; 62(1):10-24. 